\title{
A model based on two-dimensional shear wave elastography for acute-on-chronic liver failure development in patients with acutely decompensated hepatitis B cirrhosis
}

\author{
Songsong Yuan ${ }^{1 \#}$, Xingzhi Huang ${ }^{2 \#}$, Xiaoping $\mathrm{Wu}^{1}, \mathrm{Pan} \mathrm{Xu}^{2}$, Aiyun Zhou ${ }^{2}$ \\ ${ }^{1}$ Department of Infectious Disease, the First Affiliated Hospital of Nanchang University, Nanchang, China; ${ }^{2}$ Department of Ultrasonography, the \\ First Affiliated Hospital of Nanchang University, Nanchang, China \\ Contributions: (I) Conception and design: S Yuan, X Huang, P Xu, A Zhou; (II) Administrative support: X Wu, S Yuan, P Xu, A Zhou; (III) Provision \\ of study materials or patients: X Wu, S Yuan; (IV) Collection and assembly of data: X Huang, P Xu; (V) Data analysis and interpretation: X Huang, $\mathrm{P}$ \\ $\mathrm{Xu}$; (VI) Manuscript writing: All authors; (VII) Final approval of manuscript: All authors. \\ \#These authors contributed equally to this work.
}

Correspondence to: Songsong Yuan, MM. Department of Infectious Disease, the First Affiliated Hospital of Nanchang University, No. 17, Yongwaizheng Road, Donghu District, Nanchang 330006, China. Email: yuansong5255@163.com; Pan Xu, MM; Prof. Aiyun Zhou. Department of Ultrasonography, the First Affiliated Hospital of Nanchang University, No. 17, Yongwaizheng Road, Donghu District, Nanchang 330006, China. Email: xupan_1989@126.com; zhouaiyun1960@163.com.

Background: To evaluate the accuracy of two-dimensional (2D) shear wave elastography (SWE), develop and validate a novel prognostic model in predicting acute-on-chronic liver failure (ACLF) development in patients with acutely decompensated hepatitis B cirrhosis.

Methods: This prospective cohort study enrolled 221 patients in the First Affiliated Hospital of Nanchang University from September 2019 to January 2021, and randomly assigned them to the derivation and validation cohorts (7:3 ratio). Ultrasound, 2D SWE, clinical and laboratory data were collected, and outcome (ACLF developed) was recorded during a 90-day follow-up period. We evaluated the ability of 2D SWE to predict the outcome, developed a model for predicting ACLF development in the derivation cohort, and assessed the model in the validation cohort.

Results: 2D SWE values were significantly higher in patients with ACLF development $(\mathrm{P}<0.05)$. The accuracy of 2D SWE in predicting the outcome was better than that of serum parameters of liver fibrosis (all $\mathrm{P}<0.05)$. The SWE model for ACLF development had good calibration and discrimination [concordance index (C-index): 0.855 and 0.840 respectively] in derivation and validation cohorts, outperforming serum prognostic scores (all $\mathrm{P}<0.05$ ).

Conclusions: The SWE model, superior to serum prognostic scores in predicting ACLF development, could be a noninvasive tool to guide the individual management of patients with acutely decompensated hepatitis B cirrhosis.

Keywords: Acute-on-chronic liver failure (ACLF); hepatitis B virus; acute decompensation (AD); shear wave elastography (SWE)

Submitted Sep 01, 2021. Accepted for publication Jan 14, 2022.

doi: 10.21037/qims-21-871

View this article at: https://dx.doi.org/10.21037/qims-21-871 


\section{Introduction}

Chronic hepatitis B viral (HBV) infection is a global health problem, especially in Asia, where HBV is a significant cause of cirrhosis and liver-related death $(1,2)$. Acute decompensation (AD) of liver cirrhosis is the acute development of infection, ascites, gastrointestinal hemorrhage, hepatic encephalopathy, or any combination thereof $(3,4)$. A subset of patients with $\mathrm{AD}$ may be at risk of acute-on-chronic liver failure (ACLF), which is characterized by the presence of organ failure and severe systemic inflammation, and the 28 -day mortality rate in patients with ACLF in the 2013 CANONIC study was as high as $34 \%(5,6)$.

The 2020 PREDICT study by the European Association for the Study of the Liver-Chronic Liver Failure Consortium (CLIF-C) showed that pre-ACLF patients, developed ACLF within 90 days of enrollment, are significantly related to severe systemic inflammation, ACLF development and 90-day mortality (7). While identifying patients who will develop ACLF is of utmost clinical importance, the predictive performance of the Child-Pugh, model for end-stage liver disease (MELD), MELD-Na, CLIF-C AD, and ACLF-D scores are unsatisfactory (7). Moreover, the latter two models were developed using data from the European population, where alcohol and hepatitis $\mathrm{C}$ virus are predominant causes of liver cirrhosis.

Liver stiffness measurement (LSM) using shear wave elastography (SWE) is correlated with fibrosis, inflammation and necrosis. LSM has recently gained increased attention as a prognostic predictor for cirrhosis patients (8-11). Two-dimensional (2D) SWE is increasingly used due to its higher success rate of measurement and comparable accuracy compared to transient elastography (TE) (11). 2D SWE can be applied to patients with ascites, and performed with B-mode image guidance. Previous studies $(9,10,12,13)$ determined that 2D SWE values were significantly associated with liver fibrosis, liver-related events and prognosis. Few prior studies used 2D SWE to predict ACLF development among patients with acutely decompensated hepatitis B cirrhosis (AD-HBV).

Our purposes were: (I) to evaluate the accuracy of 2D SWE and (II) to develop and validate a model in predicting 90-day ACLF development for patients with AD-HBV. We present the following article in accordance with the TRIPOD reporting checklist (available at https://qims. amegroups.com/article/view/10.21037/qims-21-871/rc).

\section{Methods}

\section{Study population}

The local medical ethics committee approved the prospective cohort study of the hospital (No. 2019024). The study was conducted in accordance with the Declaration of Helsinki (as revised in 2013). Written informed consent was obtained from each patient.

Patients with AD-HBV hospitalized at the Department of Infectious Diseases of the First Affiliated Hospital of Nanchang University from September 2019 to January 2021 were enrolled. Patients were excluded if they had alcohol-, autoimmunity-, toxicity-, or drug-induced liver damage, hepatocellular carcinoma, severe chronic extrahepatic diseases, HIV infection, immunosuppressive therapy, or unqualified 2D SWE images. Patients were randomly allocated to the derivation and validation cohorts at a ratio of 7:3. Diagnostic criteria for $\mathrm{AD}$ were based on the development of ascites, hepatic encephalopathy, gastrointestinal hemorrhage, infection, or any combination of these. ACLF was diagnosed according to the CANONIC study criteria (5).

The start date of the follow-up period was the date of $\mathrm{AD}$ diagnosis. Each enrolled patient was given standard medical treatments. All patients were monitored for at least 90 days. The outcome (ACLF developed) of each patient was recorded.

\section{Baseline data and prognostic scores calculation}

The baseline clinical data, including infection (spontaneous peritonitis, chest infection, lung infection, and sepsis diagnosed within the 48-hour period that preceded the onset of $\mathrm{AD}$ ) (14), ascites, splenomegaly, upper gastrointestinal bleeding, hepatic encephalopathy, and laboratory data were recorded. Two serum parameters of liver fibrosis [aspartate aminotransferase-to-platelet ratio index (APRI) and fibrosis-4 index] were calculated $(15,16)$. Prognostic scores including Child-Pugh, MELD and MELD-Na scores were calculated as well. The formulae see Appendix 1.

\section{Conventional ultrasonographic examination}

Spleen longitudinal diameter and portal vein diameter were recorded. The peak velocity of portal vein $(\mathrm{PVv})$, hepatic arterial velocity and hepatic arterial resistive index were 

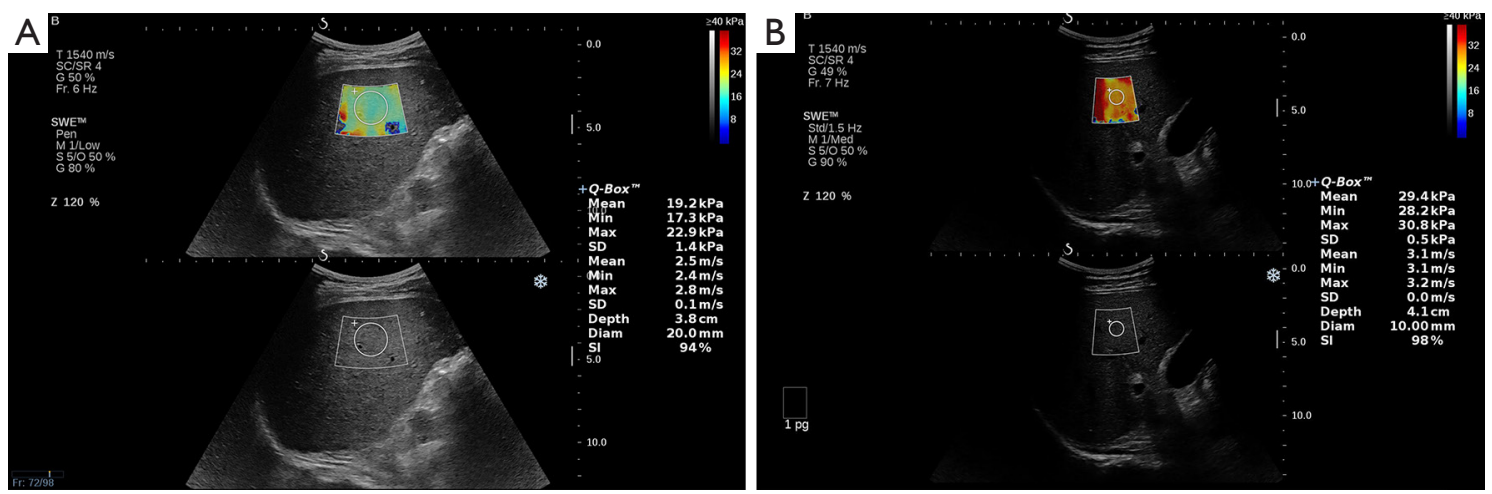

Figure 1 Image shows liver stiffness measured with 2D SWE in patients with acutely decompensated hepatitis B cirrhosis. The rectangular elasticity box $(4 \mathrm{~cm} \times 3 \mathrm{~cm})$ was placed $1-2 \mathrm{~cm}$ under the liver capsule in the parenchyma area free of large vessels. Regions of interest ranged from 10 to $20 \mathrm{~mm}$ in diameter and were positioned in the elasticity box. 2D, two-dimensional; SWE, shear wave elastography.

measured using a doppler angle of less than or equal to $60^{\circ}$ for angle correction (17).

\section{D SWE examination}

The 2D SWE procedure was performed by two radiologists with more than 5 years of ultrasonic experience using an Aixplorer US system (SuperSonic Imagine, Aix-enProvence, France) equipped with an SC6-1 convex probe. Two operators were unaware of clinical information and strictly followed the guidelines of the Society of Radiologists in Ultrasound (Figure 1) (18). Patients fasted for more than 4 hours before the imaging procedure. The 2D SWE value was defined as the median value of five measurements. Reliability of the measurement was assessed by the interquartile range/median ratio (IQR/M): "very reliable" (IQR/M $\leq 0.10)$, "reliable" $(0.10<\mathrm{IQR} / \mathrm{M} \leq 0.30)$, and "poorly reliable" (IQR/M>0.30) referring to TE assessment (8). A measurement was considered acceptable if the IQR/M $\leq 0.30$ and more than two-thirds of the signal obtained in the elasticity box.

\section{Statistical analysis}

Statistical tests were performed by using SPSS Statistics software (version 23; IBM, New York, NY, USA) and R software (version 4.0.1, https://www.r-project.org/; packages used are provided in Table S1). The Shapiro-Wilk test was used to evaluate the normal distribution. Continuous data were expressed as means \pm standard deviation or M (IQR) and compared using Student's $t$-test or Mann-Whitney
$\mathrm{U}$ test. Categorical data were expressed as the $\mathrm{n}(\%)$ and compared using a chi-square test or Fisher's exact test.

For the derivation group, baseline factors significantly associated with 90-day ACLF development $(\mathrm{P}<0.05)$ in the Fine-Gray test were entered in the multivariable analysis by using the proportional-hazards competing risk (PHCR) model, considering death and liver transplantation as competing risks. Parameters significantly associated with the outcome $(\mathrm{P}<0.05)$ and with a limited internal colinearity (variance inflation factor $\leq 10$ ) were used to fit the new model. The performance of the 2D SWE and new model were compared with serum parameters of liver fibrosis and prognostic scores in the validation and entire cohorts, respectively. Calibration performance was evaluated using a calibration curve and the Hosmer-Lemeshow test; discrimination performance was evaluated using Harrell's concordance index (C-index). Integrated Discriminating Improvement (IDI) statistic was used to compare C-index (19). A confirmatory analysis was carried out to assess the discrimination ability of the new model by the area under the receiver operating characteristic curve (AUC) for 90-day ACLF development. Comparisons of AUCs were assessed by using the DeLong test.

The thresholds for 2D SWE values and the new model scores were defined as the value that produced the largest $\chi^{2}$ value in the Mantel-Cox test (20). Survival probability (Kaplan-Meier) curves were constructed for different groups and compared using a log-rank test. A nomogram was drawn up based on the parameters used as categorical variables. The discrimination and calibration were assessed. The statistical significance level was set at $\mathrm{P}<0.05$. 


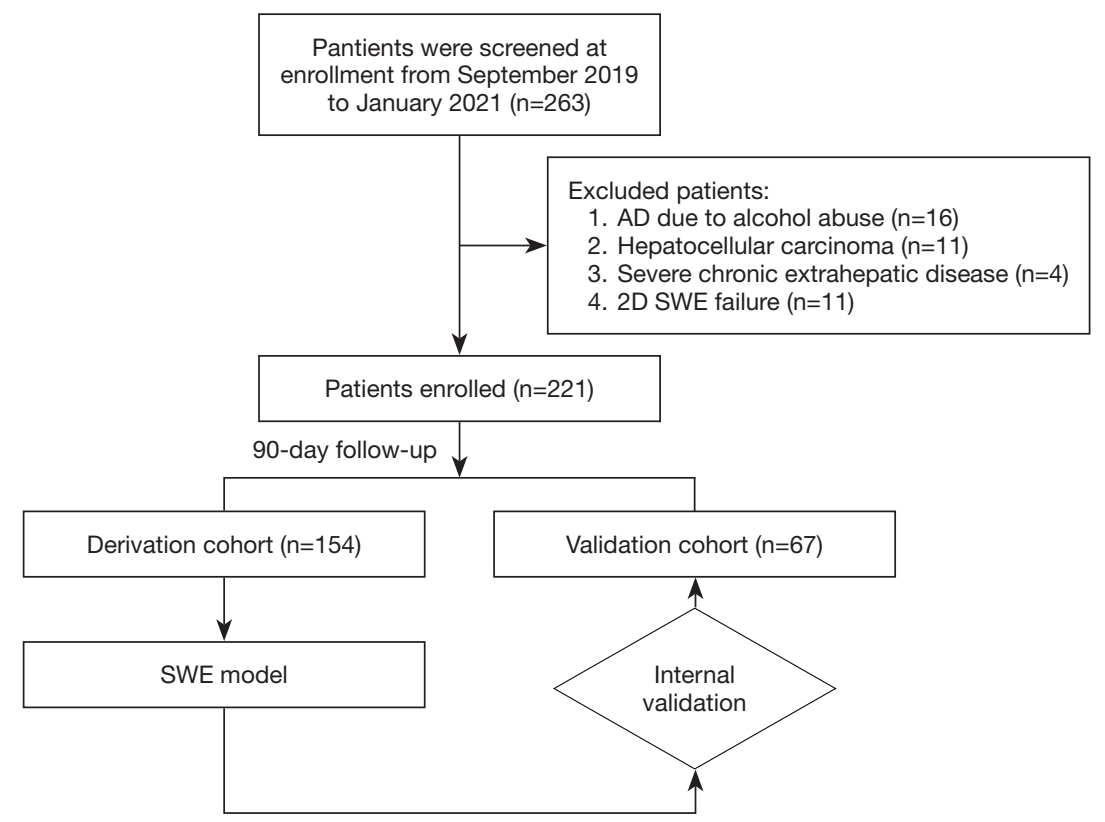

Figure 2 Flowchart shows patient enrollment in the study. AD, acute decompensation; 2D, two-dimensional; SWE, shear wave elastography.

\section{Results}

\section{Patient characteristics}

A total of $263 \mathrm{AD}-\mathrm{HBV}$ patients met the inclusion criteria, and 221 were eventually enrolled (154 in the derivation cohort and 67 in the validation cohort) (Figure 2). Baseline characteristics are shown in Table 1. Among the derivation cohort, 33 patients developed ACLF, 4 deceased without ACLF development, and 2 received liver transplantation; 17 developed ACLF, 2 deceased without ACLF development, and 1 received transplantation among the validation cohort. There was a significant difference in frequency of hepatic encephalopathy, levels of age, g-glutamyl transferase and hepatic arterial velocity among the two cohorts (all $\mathrm{P}<0.05$ ). According to the outcome, the distribution of clinical and laboratory characteristics are shown in Table S2. Patients with 90-day ACLF development had significantly lower serum sodium and prothrombin activity (PTA) levels and significantly higher prothrombin times, international normalized ratio, MELD, MELD-Na, and Child-Pugh levels than patients without ACLF development (all $\mathrm{P}<0.05$ ).

\section{Predictive power of $2 D S W E$}

The success rate of $2 \mathrm{D}$ SWE in patients was $95.3 \%$ (11/232). $26.3 \%(61 / 232)$ of the 2D SWE results were evaluated as "very reliable", and $69.0 \%(160 / 232)$ were considered as "reliable". 2D SWE failed in 11 patients due to liver atrophy $(\mathrm{n}=3)$, intestinal gas or obesity $(\mathrm{n}=6)$, or inability to hold their breath $(\mathrm{n}=2)$. 2D SWE values were significantly higher in patients with ACLF development (derivation cohort: 44.8 vs. $26.2 \mathrm{kPa}, \mathrm{P}<0.001$; validation cohort: 42.8 vs. $24.2 \mathrm{kPa}, \mathrm{P}=0.001)$. Furthermore, 2D SWE was a risk factor for 90-day ACLF development (HR $=1.050 ; 95 \%$ CI: $1.037-1.063 ; \mathrm{P}<0.001)$. The C-index in the derivation $(0.777 ; 95 \%$ CI: $0.706-0.848)$ and validation (0.754, 95\% CI: $0.638-0.870)$ cohorts were significantly better than those corresponding to the APRI and fibrosis-4 index (Table 2). The AUC of 2D SWE in the entire cohort (0.794; 95\% CI: 0.724-0.865) was significantly higher than the other (Figure $3 A$ ).

\section{Development of SWE model in the derivation cohort}

Baseline factors significantly associated with ACLF developed for derivation cohort were age, white-cell count, serum sodium, bilirubin, alkaline phosphatase (ALP), prothrombin times, international normalized ratio, PTA, $\mathrm{PVv}$ and 2D SWE values in univariate analysis. After fitting an initial CR-PH model with all these factors, serum sodium, ALP, PTA, PVv and 2D SWE values were selected as the best predictors (Table 3). The most significant effect size was observed at 2D SWE values (1.141), followed by 
Table 1 Baseline characteristics of patients in the derivation and validation cohorts

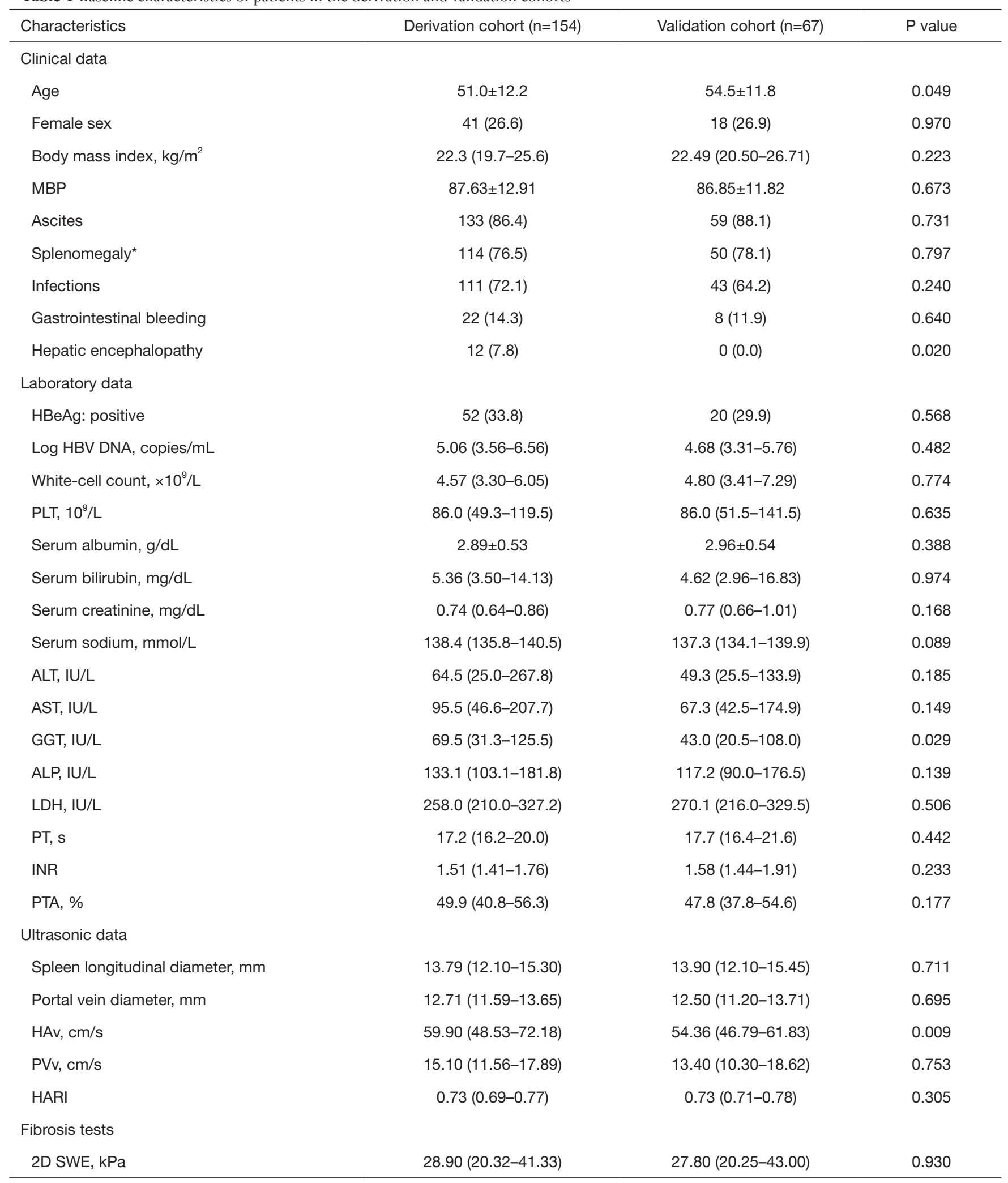

Table 1 (continued) 
Table 1 (continued)

\begin{tabular}{|c|c|c|c|}
\hline Characteristics & Derivation cohort $(n=154)$ & Validation cohort $(n=67)$ & $P$ value \\
\hline \multicolumn{4}{|l|}{ SWE results } \\
\hline "Very reliable"\#" & $43(26.5)$ & $18(25.7)$ & 0.965 \\
\hline "Reliable"\#" & $111(68.5)$ & $49(70.0)$ & \\
\hline "Poorly reliable"" & $8(4.9)$ & $3(4.3)$ & \\
\hline Fibrosis-4 index & $7.51(4.56-10.93)$ & $6.55(4.52-12.79)$ & 0.880 \\
\hline \multicolumn{4}{|l|}{ Severity scores } \\
\hline Child-Pugh & $12(11-13)$ & $12(11-13)$ & 0.665 \\
\hline MELD & $18.33(15.42-22.84)$ & $18.77(15.01-24.15)$ & 0.615 \\
\hline \multicolumn{4}{|l|}{ Outcome } \\
\hline 90-day ACLF development rate & $33(21.4)$ & $17(25.4)$ & 0.519 \\
\hline
\end{tabular}

Continuous data were expressed as mean \pm standard deviation or median (25-75\% quantiles); categorical data were expressed as $\mathrm{n}(\%)$. ${ }^{\text {, }}$ 5 patients of the derivation cohort and 3 of the validation cohort underwent splenectomy; ", 2D SWE reliability by IQR/M: "very reliable" (IQR/ $M \leq 0.10)$, "reliable" (0.10<IQR/M $\leq 0.3)$, and "poorly reliable" (IQR/M >0.30). MBP, mean arterial pressure; HBeAg, hepatitis B e antigen; HBV, hepatitis B viral; PLT, platelet count; ALT, alanine aminotransferase; AST, aspartate aminotransferase; GGT, g-glutamyl transferase; ALP, alkaline phosphatase; LDH, lactate dehydrogenase; PT, prothrombin time; INR, international normalized ratio; PTA, prothrombin activity; HAv, hepatic arterial velocity; PVv, peak velocity of portal vein; HARI, hepatic arterial resistive index; 2D, two-dimensional; SWE, shear wave elastography; APRI, aspartate aminotransferase-to-platelet ratio index; MELD, model for end-stage liver disease; ACLF, acuteon-chronic liver failure; IQR/M, interquartile range/median ratio.

Table 2 Predictive ability of 2D SWE as compared to serum biomarkers of liver fibrosis

\begin{tabular}{|c|c|c|c|}
\hline Cohorts & 2D SWE, C-index (95\% Cl) & APRI, C-index (95\% Cl) & Fibrosis-4 index, C-index (95\% Cl) \\
\hline 90-day ACLF development & $0.777(0.706-0.848)$ & $0.620(0.528-0.712)$ & $0.540(0.434-0.646)$ \\
\hline$P$ value vs. SWE model ${ }^{*}$ & - & 0.046 & 0.004 \\
\hline \multicolumn{4}{|l|}{ Validation cohort $(n=67)$} \\
\hline$P$ value vs. SWE model ${ }^{*}$ & - & 0.014 & 0.028 \\
\hline
\end{tabular}

*, $P$ values from the IDI statistics test. 2D, two-dimensional; SWE, shear wave elastography; C-index, concordance index; APRI, aspartate aminotransferase-to-platelet ratio index; ACLF, acute-on-chronic liver failure; IDI, Integrated Discriminating Improvement.

PTA (0.964) (Figure S1). The formula of the SWE model according to the CR-PH model was presented as follow:

$$
\begin{aligned}
\text { SWE model score }= & 0.03 \times 2 \operatorname{DSWE}(\mathrm{kPa})-0.062 \times \operatorname{sodium}(\mathrm{mmol} / \mathrm{L}) \\
& +0.003 \times A L P(I \mathrm{IU} / \mathrm{L})-0.043 \times P T A(\%) \\
& -0.099 \times P V v(\mathrm{~cm} / \mathrm{s})+10
\end{aligned}
$$

In our series, the extreme SWE model scores were from -3.652 to 2.746. Patients with 90-day ACLF development had significantly higher SWE model scores than patients without ACLF development in the derivation (0.37 vs. -1.16; $\mathrm{P}<0.001)$ and validation $(0.55$ vs. $-1.12 ; \mathrm{P}<0.001)$ cohorts. The Hosmer-Lemeshow test statistic $\left(\chi^{2}=3.676 ; \mathrm{P}=0.885\right)$ and calibration curve showed good calibration in the derivation cohort (Figure S2). The C-Index of the SWE model (0.855; $95 \%$ CI: $0.810-0.900)$ was significantly better than that of MELD, MELD-Na, and Child-Pugh scores (Table 4). 

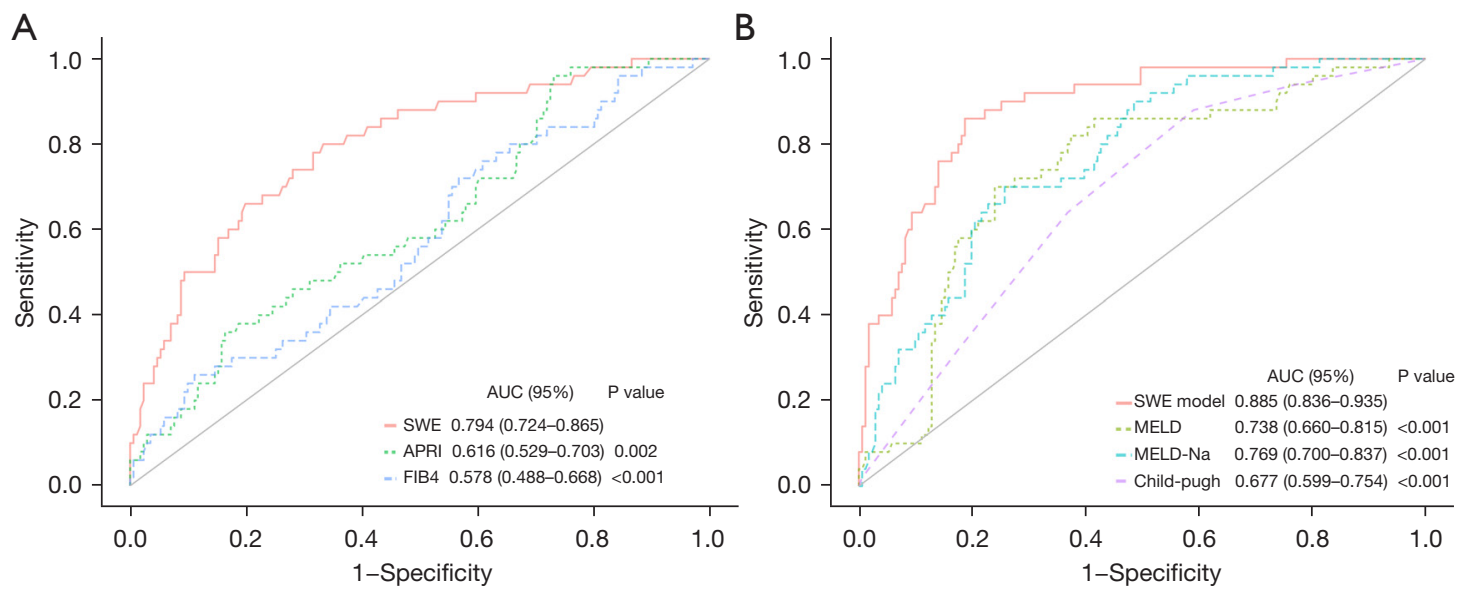

Figure 3 Receiver operating characteristic curves shows that predictive performance of 2D SWE values (A) and SWE model scores (B) are compared with serum biomarkers of liver fibrosis and prognostic models for 90-day ACLF development. 2D, two-dimensional; SWE, shear wave elastography; ACLF, acute-on-chronic liver failure; APRI, aspartate aminotransferase-to-platelet ratio index; FIB4, fibrosis-4 index; MELD, model for end-stage liver disease; AUC, area under the receiver operating characteristic curve.

Table 3 Univariate and multivariate PH-CR model of independent risk factors for 90-day ACLF development

\begin{tabular}{|c|c|c|c|c|c|c|}
\hline Characteristics & \multicolumn{3}{|c|}{ Univariate } & \multicolumn{3}{|c|}{ Multivariate } \\
\hline Age & 0.972 & $0.949-0.996$ & 0.024 & & & \\
\hline White-cell count, $\times 10^{9} / \mathrm{L}$ & 1.110 & $1.010-1.230$ & 0.029 & & & \\
\hline Serum bilirubin, mg/dL & 1.060 & $1.031-1.089$ & $<0.001$ & & & \\
\hline ALP, IU/L & 1.003 & $0.998-1.002$ & 0.019 & 1.003 & $1.001-1.005$ & 0.009 \\
\hline $\mathrm{PT}, \mathrm{s}^{*}$ & 1.060 & $1.010-1.120$ & 0.015 & & & \\
\hline $\mathrm{INR}^{*}$ & 3.260 & $2.592-3.928$ & 0.001 & & & \\
\hline PTA, \% & 0.942 & $0.918-0.966$ & $<0.001$ & 0.958 & $0.927-0.989$ & 0.008 \\
\hline
\end{tabular}

*, the variance inflation factors of PT and INR were 10.173 and 11.296. PH-CR, proportional-hazards competing risk; ACLF, acute-onchronic liver failure; ALP, alkaline phosphatase; PT, prothrombin time; INR, international normalized ratio; PTA, prothrombin activity; PVv, peak velocity of portal vein; 2D, two-dimensional; SWE, shear wave elastography.

\section{Validation of the SWE model}

For the validation cohort, the C-index of the SWE model (0.840; 95\% CI: 0.746-0.934) was significantly better than that of Child-Pugh, MELD and MELD-Na scores (Table 4). The AUC $(0.885 ; 95 \%$ CI: 0.836-0.935) confirmed the superiority (Figure 3B). Moreover, the percentage improvement obtained with the SWE model in prediction error rate for the prognostic scores $[100 \times(\mathrm{C}$-index SWE model - C-index REF)/(1 - C-index REF)] were from $33.9 \%$ to $53.3 \%$ (Figure $\mathrm{S} 3$ ).

\section{Development of the SWE nomogram}

Cutoff values were 29.2 for 2D SWE values, $138.4 \mathrm{mmol} / \mathrm{L}$ for serum sodium, 106 IU/L for ALP, $40.2 \%$ for PTA and 
Table 4 Predictive ability of the SWE model as compared to prognostic scores

\begin{tabular}{lcccc}
\hline Cohorts & $\begin{array}{c}\text { SWE model, C-index } \\
(95 \% \mathrm{Cl})\end{array}$ & $\begin{array}{c}\text { Child-Pugh, C-index } \\
(95 \% \mathrm{Cl})\end{array}$ & $\begin{array}{c}\text { MELD, C-index } \\
(95 \% \mathrm{Cl})\end{array}$ & $\begin{array}{c}\text { MELD-Na, C-index } \\
(95 \% \mathrm{Cl})\end{array}$ \\
\hline $\begin{array}{l}\text { Derivation cohort }(\mathrm{n}=154) \\
\text { 90-day ACLF development }\end{array}$ & $0.855(0.810-0.900)$ & $0.742(0.666-0.818)$ & $0.746(0.677-0.815)$ & $0.664(0.578-0.750)$ \\
P value vs. SWE model & - & 0.002 & $<0.001$ \\
Validation cohort $(\mathrm{n}=67)$ & & & 0.001 \\
90-day ACLF development & $0.840(0.746-0.934)$ & $0.724(0.618-0.830)$ & $0.764(0.662-0.866)$ & $0.666(0.550-0.782)$ \\
P value vs. SWE model & - & $<0.001$ & 0.010 \\
\hline
\end{tabular}

*, $\mathrm{P}$ values from the IDI statistics test. SWE, shear wave elastography; C-index, concordance index; MELD, model for end-stage liver disease; ACLF, acute-on-chronic liver failure; IDI, Integrated Discriminating Improvement.

$12.8 \mathrm{~cm} / \mathrm{s}$ for PVv. The cutoff values for the 2D SWE and SWE model were used to separate patients into lowrisk and high-risk groups. Patients in high-risk groups had a significantly higher risk for ACLF development in the validation and entire cohorts (Figure 4). A nomogram incorporating the five predictors mentioned above, used as categorical variables, was constructed (Figure 5A). The calibration curve and Hosmer-Lemeshow test statistic $(\mathrm{P}=0.405$ and 0.600 respectively) showed satisfactory calibration in the derivation and validation cohorts (Figure 5B, 5C). The C-index of 0.817 (95\% CI: 0.764-0.870) in the derivation cohort and 0.836 (95\% CI: $0.740-0.932$ ) in the validation cohort demonstrated good discrimination.

\section{Discussion}

Our results showed that 2D SWE was correlated with 90-day ACLF development in patients with AD-HBV, whose predictive ability was better than APRI and fibrosis-4 index. The SWE model, composed of serum sodium, ALP, PTA, PVv and 2D SWE values, showed good calibration and discrimination. The novel model improved the prediction of Child-Pugh, MELD and MELD-Na scores and could aid the management of $\mathrm{AD}-\mathrm{HBV}$ patients.

The PREDICT study found that the pre-ACLF is a specific course related to worse prognosis in patients with $\mathrm{AD}$, indicating that rapid progression of systemic inflammation and ACLF development and short-term mortality, of which 90-day mortality rate was $53.7 \%$ (7). The different pathophysiological mechanisms and their corresponding effects on the liver make LSM possible to predict ACLF development (7). The most effective treatment of ACLF is liver transplantation, while it is not an available treatment for most patients. Therefore, antiviral therapy (interferon, nucleoside analogs) and artificial liver support systems (ALSSs) are the main alternatives to liver transplants; hence the mortality of patients with ACLF remains high $(21,22)$. Serum prognostic scores for evaluating the prognosis of liver cirrhosis have limitations and were not the target for patients with AD-HBV.

LSM can be affected by inflammation, necrosis, and liver function $(8,23,24)$. Qiu et al. (25) suggested that LSM was significantly related to liver function reserve. LSM is also associated with portal hypertension, supporting that it can reflect the clinical course of AD. A meta-analysis (26) showed that LSM was significantly related to liverrelated events, including decompensation, hepatocellular carcinoma, and death. The prediction for the liver failure of LSM was comparable with that of MELD (27-30).

LSM tools, including TE, point SWE and 2D SWE, are now widely used. TE was recommended as a first-line examination for liver fibrosis and cirrhosis (31). Previous studies found that 2D SWE had comparable diagnostic accuracy to TE $(8,11,12)$. Moreover, it is inappropriate for hospitalized patients with liver cirrhosis to perform TE because of ascites and liver atrophy. 2D SWE has a success rate of $86 \%-100 \%$ in patients with ascites (10). Our study confirmed excellent success rates of 2D SWE in patients with $\mathrm{AD}-\mathrm{HBV}$, whose images were successfully captured in $95.3 \%(221 / 232)$ of patients. Furthermore, when measuring 2D SWE values, we can simultaneously measure $\mathrm{PVv}$ and monitor ascites and liver tumors. $\mathrm{Wu}$ et al. (12) demonstrated that 2D SWE could predict liverrelated events in patients with compensated liver cirrhosis. Jin et al. (10) found that 2D SWE improved the predictive performance of MELD in ACLF patients. Patients with 

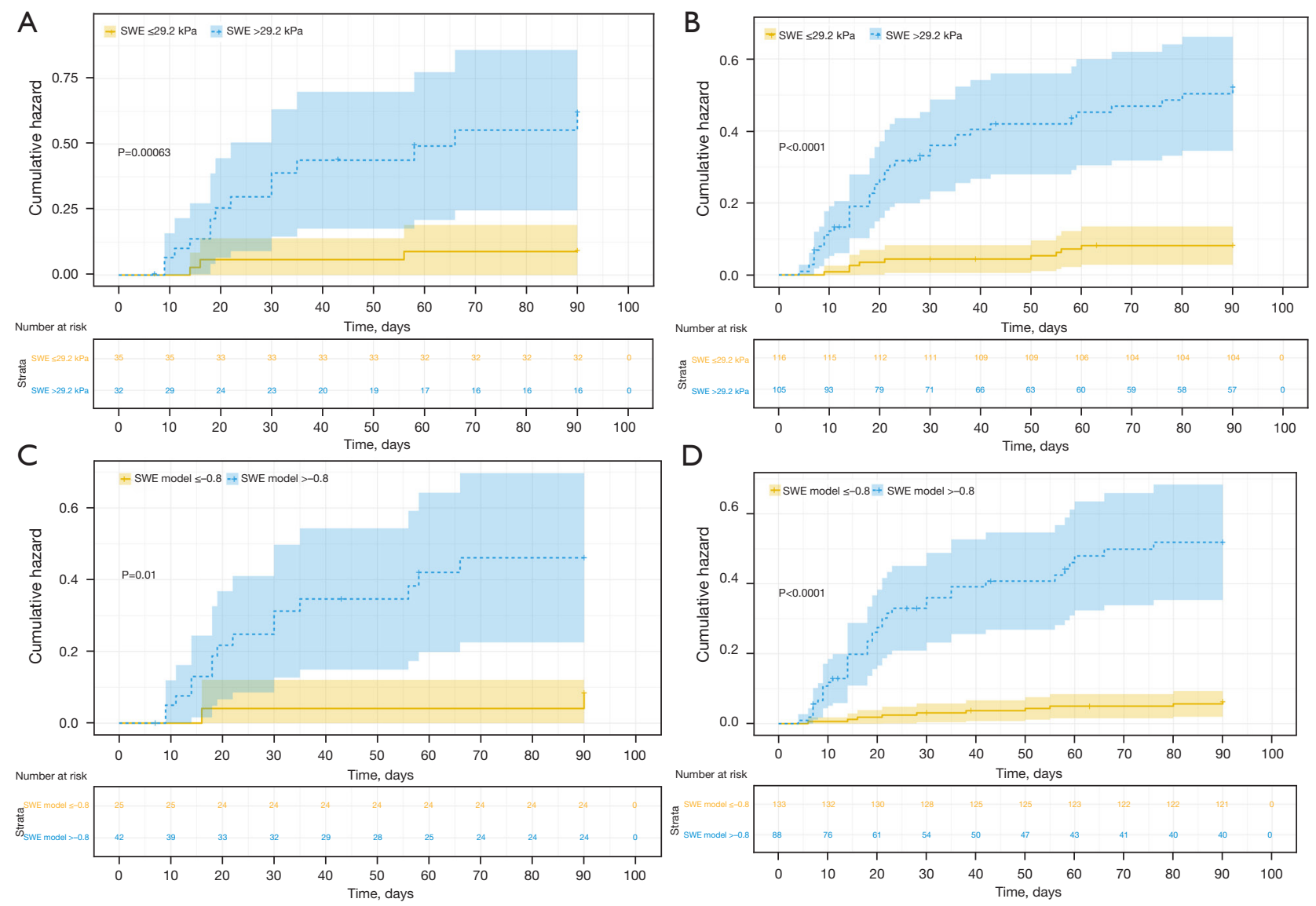

Figure 4 Kaplan-Meier 90-day ACLF development analyses based on the 2D SWE value and SWE model for patients with acutely decompensated hepatitis B cirrhosis in the validation and entire cohorts. 90-day ACLF development probability was significantly higher in patients with high 2D SWE values $(>29.2 \mathrm{kPa})$ than in patients with low 2D SWE values $(\leq 29.2 \mathrm{kPa})$ in the validation $(\mathrm{A})$ and entire cohorts (B); 90-day ACLF development probability was significantly higher in patients with high SWE model score (>-0.8) than in patients with low SWE model score $(\leq-0.8)$ in the validation $(C)$ and entire cohorts (D). ACLF, acute-on-chronic liver failure; 2D, two-dimensional; SWE, shear wave elastography.

higher 2D SWE values are more likely to have worse outcomes (27-30). In our study, 90-day ACLF development was correlated with higher 2D SWE values. The predictive performance of 2D SWE was better than that of APRI and fibrosis-4 index. Meanwhile, 2D SWE had the largest effect size in our SWE model. Given the above, 2D SWE could be a noninvasive and accurate predictor in patients with different stages of chronic liver disease.

The SWE model developed in our study contained parameters that can represent LSM, liver blood flow, electrolytes, coagulation and liver function. These parameters are usually involved in the systematic and prognostic evaluation. Our model was better than the Child-Pugh, MELD and MELD-Na scores to provide prognostic information and accurately stratify patients into high and low-risk groups. A nomogram composed of these parameters used as categorical variables is based on a multivariate Cox proportional hazards model and displays the probable value of individual ACLF development concisely and intuitively. Our nomogram also showed favorably predictive performance; hence it may effectively assist individualized treatment.

Our study had several limitations. First, this study was performed in a single center with small sample size; hence, further studies with multiple centers and larger samples are needed. Second, further research is necessary to evaluate the influence of the etiology, antiviral therapy and pathological inflammation grade for prediction. Third, we did not 


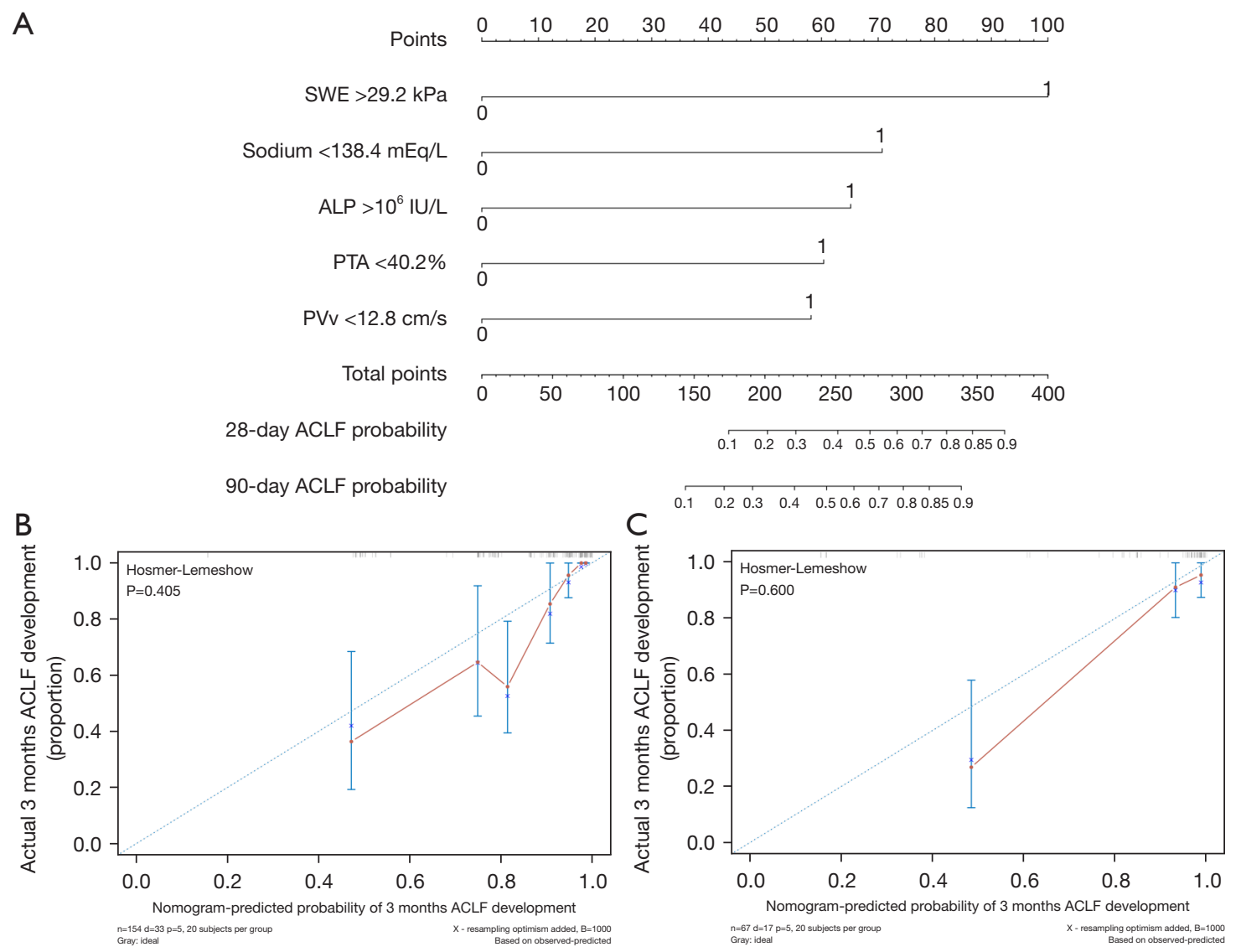

Figure 5 Generation and evaluation of the nomogram. (A) Nomogram to predict 90-day ACLF development; (B,C) the calibration curve estimates 90-day ACLF development predicted by nomogram in the derivation and validation cohorts. ACLF, acute-on-chronic liver failure; ALP, alkaline phosphatase; PTA, prothrombin activity, PVv, peak velocity of portal vein; SWE, shear wave elastography.

analyze the dynamic changes in 2D SWE values and SWE model scores of patients for the possible better presence of the correlation between parameters and prognosis.

\section{Conclusions}

2D SWE is a noninvasive and promising predictor for 90-day ACLF development in patients with AD-HBV. The prognostic model based on 2D SWE values, developed by our study, could be an individualized tool and provide a more accurate prognosis prediction than serum prognostic scores. Patients with higher 2D SWE values and SWE model scores may benefit from intensive medical treatment.

\section{Acknowledgments}

Funding: This study was supported by Natural
Science Foundation of Jiangxi Province of China (No. 20212ACB206010).

\section{Footnote}

Reporting Checklist: The authors have completed the TRIPOD reporting checklist. Available at https://qims. amegroups.com/article/view/10.21037/qims-21-871/rc

Conflicts of Interest: All authors have completed the ICMJE uniform disclosure form (available at https://qims. amegroups.com/article/view/10.21037/qims-21-871/coif). The authors have no conflicts of interest to declare.

Ethical Statement: The authors are accountable for all aspects of the work in ensuring that questions related to the accuracy or integrity of any part of the work are 
appropriately investigated and resolved. The study was conducted in accordance with the Declaration of Helsinki (as revised in 2013). The study was approved by local medical ethics committee of the First Affiliated Hospital of Nanchang University (No. 2019024) and informed consent was taken from all individual participants.

Open Access Statement: This is an Open Access article distributed in accordance with the Creative Commons Attribution-NonCommercial-NoDerivs 4.0 International License (CC BY-NC-ND 4.0), which permits the noncommercial replication and distribution of the article with the strict proviso that no changes or edits are made and the original work is properly cited (including links to both the formal publication through the relevant DOI and the license). See: https://creativecommons.org/licenses/by-nc-nd/4.0/.

\section{References}

1. Stanaway JD, Flaxman AD, Naghavi M, Fitzmaurice C, Vos T, Abubakar I, et al. The global burden of viral hepatitis from 1990 to 2013: findings from the Global Burden of Disease Study 2013. Lancet 2016;388:1081-8.

2. Zhang S, Wang F, Zhang Z. Current advances in the elimination of hepatitis B in China by 2030. Front Med 2017;11:490-501.

3. Fukui H, Saito H, Ueno Y, Uto H, Obara K, Sakaida I, Shibuya A, Seike M, Nagoshi S, Segawa M, Tsubouchi H, Moriwaki H, Kato A, Hashimoto E, Michitaka K, Murawaki T, Sugano K, Watanabe M, Shimosegawa T. Evidence-based clinical practice guidelines for liver cirrhosis 2015. J Gastroenterol 2016;51:629-50.

4. Trebicka J, Fernandez J, Papp M, Caraceni P, Laleman W, Gambino C, et al. PREDICT identifies precipitating events associated with the clinical course of acutely decompensated cirrhosis. J Hepatol 202 1;74:1097-108.

5. Moreau R, Jalan R, Gines P, Pavesi M, Angeli P, Cordoba J, Durand F, Gustot T, Saliba F, Domenicali M, Gerbes A, Wendon J, Alessandria C, Laleman W, Zeuzem S, Trebicka J, Bernardi M, Arroyo V; CANONIC Study Investigators of the EASL-CLIF Consortium. Acute-on-chronic liver failure is a distinct syndrome that develops in patients with acute decompensation of cirrhosis. Gastroenterology 2013;144:1426-37, 1437.e1-9.

6. Jalan R, Saliba F, Pavesi M, Amoros A, Moreau R, Ginès $\mathrm{P}$, et al. Development and validation of a prognostic score to predict mortality in patients with acute-on-chronic liver failure. J Hepatol 2014;61:1038-47.
7. Trebicka J, Fernandez J, Papp M, Caraceni P, Laleman W, Gambino C, et al. The PREDICT study uncovers three clinical courses of acutely decompensated cirrhosis that have distinct pathophysiology. J Hepatol 2020;73:842-54.

8. Gao Y, Zheng J, Liang P, Tong M, Wang J, Wu C, et al. Liver Fibrosis with Two-dimensional US Shear-Wave Elastography in Participants with Chronic Hepatitis B: A Prospective Multicenter Study. Radiology 2018;289:407-15.

9. Grgurević I, Bokun T, Mustapić S, Trkulja V, Heinzl R, Banić M, Puljiz Ž, Lukšić B, Kujundžić M. Real-time two-dimensional shear wave ultrasound elastography of the liver is a reliable predictor of clinical outcomes and the presence of esophageal varices in patients with compensated liver cirrhosis. Croat Med J 2015;56:470-81.

10. Jin JY, Zheng YB, Zheng J, Liu J, Mao YJ, Chen SG, Gao ZL, Zheng RQ. 2D shear wave elastography combined with MELD improved prognostic accuracy in patients with acute-on-chronic hepatitis B liver failure. Eur Radiol 2018;28:4465-74.

11. Zeng J, Zheng J, Huang Z, Chen S, Liu J, Wu T, Zheng R, Lu M. Comparison of 2-D Shear Wave Elastography and Transient Elastography for Assessing Liver Fibrosis in Chronic Hepatitis B. Ultrasound Med Biol 2017;43:1563-70.

12. Wu M, Wu L, Jin J, Wang J, Li S, Zeng J, Guo H, Zheng J, Chen S, Zheng R. Liver Stiffness Measured with Twodimensional Shear-Wave Elastography Is Predictive of Liver-related Events in Patients with Chronic Liver Disease Due to Hepatis B Viral Infection. Radiology 2020;295:353-60.

13. Yang L, Ling W, He D, Lu C, Ma L, Tang L, Luo Y, Chen S. Shear wave-based sound touch elastography in liver fibrosis assessment for patients with autoimmune liver diseases. Quant Imaging Med Surg 2021;11:1532-42.

14. Fernández J, Acevedo J, Wiest R, Gustot T, Amoros A, Deulofeu C, Reverter E, Martínez J, Saliba F, Jalan R, Welzel T, Pavesi M, Hernández-Tejero M, Ginès P, Arroyo V; European Foundation for the Study of Chronic Liver Failure. Bacterial and fungal infections in acute-onchronic liver failure: prevalence, characteristics and impact on prognosis. Gut 2018;67:1870-80.

15. Sterling RK, Lissen E, Clumeck N, Sola R, Correa MC, Montaner J, S Sulkowski M, Torriani FJ, Dieterich DT, Thomas DL, Messinger D, Nelson M; APRICOT Clinical Investigators. Development of a simple noninvasive index to predict significant fibrosis in patients with $\mathrm{HIV} / \mathrm{HCV}$ coinfection. Hepatology 2006;43:1317-25.

16. Wai CT, Greenson JK, Fontana RJ, Kalbfleisch JD, 
Marrero JA, Conjeevaram HS, Lok AS. A simple noninvasive index can predict both significant fibrosis and cirrhosis in patients with chronic hepatitis C. Hepatology 2003;38:518-26.

17. Park HS, Desser TS, Jeffrey RB, Kamaya A. Doppler Ultrasound in Liver Cirrhosis: Correlation of Hepatic Artery and Portal Vein Measurements With Model for End-Stage Liver Disease Score. J Ultrasound Med 2017;36:725-30.

18. Barr RG, Wilson SR, Rubens D, Garcia-Tsao G, Ferraioli G. Update to the Society of Radiologists in Ultrasound Liver Elastography Consensus Statement. Radiology 2020;296:263-74.

19. Gustot T, Fernandez J, Garcia E, Morando F, Caraceni $\mathrm{P}$, Alessandria C, et al. Clinical Course of acute-onchronic liver failure syndrome and effects on prognosis. Hepatology 2015;62:243-52.

20. Camp RL, Dolled-Filhart M, Rimm DL. X-tile: a new bio-informatics tool for biomarker assessment and outcome-based cut-point optimization. Clin Cancer Res 2004;10:7252-9.

21. Bernal W, Jalan R, Quaglia A, Simpson K, Wendon J, Burroughs A. Acute-on-chronic liver failure. Lancet 2015;386:1576-87.

22. Sarin SK, Kedarisetty CK, Abbas Z, Amarapurkar D, Bihari C, Chan AC, et al. Acute-on-chronic liver failure: consensus recommendations of the Asian Pacific Association for the Study of the Liver (APASL) 2014. Hepatol Int 2014;8:453-71.

23. Dietrich CF, Bamber J, Berzigotti A, Bota S, Cantisani V, Castera L, Cosgrove D, Ferraioli G, Friedrich-Rust M, Gilja OH, Goertz RS, Karlas T, de Knegt R, de Ledinghen V, Piscaglia F, Procopet B, Saftoiu A, Sidhu PS, Sporea I, Thiele M. EFSUMB Guidelines and Recommendations on the Clinical Use of Liver Ultrasound Elastography, Update 2017 (Long Version). Ultraschall Med 2017;38:e16-47.

24. Ferraioli G, Filice C, Castera L, Choi BI, Sporea I, Wilson
$\mathrm{SR}$, et al. WFUMB guidelines and recommendations for clinical use of ultrasound elastography: Part 3: liver. Ultrasound Med Biol 2015;41:1161-79.

25. Qiu T, Wang H, Song J, Guo G, Shi Y, Luo Y, Liu J. Could Ultrasound Elastography Reflect Liver Function? Ultrasound Med Biol 2018;44:779-85.

26. Singh S, Fujii LL, Murad MH, Wang Z, Asrani SK, Ehman RL, Kamath PS, Talwalkar JA. Liver stiffness is associated with risk of decompensation, liver cancer, and death in patients with chronic liver diseases: a systematic review and meta-analysis. Clin Gastroenterol Hepatol 2013;11:1573-84.e1-2; quiz e88-9.

27. Dechêne A, Sowa JP, Gieseler RK, Jochum C, Bechmann LP, El Fouly A, Schlattjan M, Saner F, Baba HA, Paul A, Dries V, Odenthal M, Gerken G, Friedman SL, Canbay A. Acute liver failure is associated with elevated liver stiffness and hepatic stellate cell activation. Hepatology 2010;52:1008-16.

28. Karlas TF, Pfrepper C, Rosendahl J, Benckert C, Wittekind C, Jonas S, Moessner J, Tröltzsch M, Tillmann HL, Berg T, Keim V, Wiegand J. Acoustic radiation force impulse (ARFI) elastography in acute liver failure: necrosis mimics cirrhosis. Z Gastroenterol 2011;49:443-8.

29. Kuroda H, Kakisaka K, Oikawa T, Onodera M, Miyamoto Y, Sawara K, Endo R, Suzuki K, Takikawa $\mathrm{Y}$. Liver stiffness measured by acoustic radiation force impulse elastography reflects the severity of liver damage and prognosis in patients with acute liver failure. Hepatol Res 2015;45:571-7.

30. Sharma P, Bansal R, Matin A, Tyagi P, Bansal N, Singla V, Kumar A, Arora A. Role of Transient Elastography (Fibroscan) in Differentiating Severe Acute Hepatitis and Acute on Chronic Liver Failure. J Clin Exp Hepatol 2015;5:303-9.

31. European Association for the Study of the Liver. EASL 2017 Clinical Practice Guidelines on the management of hepatitis B virus infection. J Hepatol 2017;67:370-98.
Cite this article as: Yuan S, Huang $\mathrm{X}, \mathrm{Wu} \mathrm{X}, \mathrm{Xu} \mathrm{P}, \mathrm{Zhou} \mathrm{A}$. A model based on two-dimensional shear wave elastography for acute-on-chronic liver failure development in patients with acutely decompensated hepatitis B cirrhosis. Quant Imaging Med Surg 2022;12(5):2732-2743. doi: 10.21037/qims-21-871 


\section{Appendix 1}

\section{Methods}

\section{Formulas for serum models of liver fibrosis or prognostic scores}

Two serum models of liver fibrosis were calculated according to the following $(15,16)$ :

APRI $=[$ AST $(\mathrm{U} / \mathrm{L}) /$ upper limit of normal AST $\times 100] /$ platelet $\operatorname{count}\left(10^{9} / \mathrm{L}\right)$

Fibrosis-4 index $=[$ age $($ year $)] \times[$ AST $(\mathrm{U} / \mathrm{L})] /\left[\right.$ platelet count $\left(10^{9} / \mathrm{L}\right) \times \operatorname{ALT}$ level $\left.(\mathrm{U} / \mathrm{L})^{1 / 2}\right]$

Three serum parameters of prognostic scores were calculated according to the following:

MELD $=[0.957 \times \ln ($ serum Cr $)+0.378 \times \ln ($ serum bilirubin $)+1.120 \times \ln ($ international normalized ratio $)+0.643] \times 10$ (if hemodialysis, value for creatinine is automatically set to 4.0 )

Note: if any score is $<1$, the MELD assumes the score is equal to 1 (32);

MELD-Na $=$ MELD score $-\mathrm{Na}-0.025 \times$ MELD $\times(140-\mathrm{Na})+140$

Note: sodium is limited in a range of $125-140$, and if outside of these bounds, is set to the nearest limit (33);

Child-Pugh (34):

\begin{tabular}{lll}
\hline Factors & Levels & Points \\
\hline Bilirubin (total) & $<2 \mathrm{mg} / \mathrm{dL}(<34.2 \mu \mathrm{mol} / \mathrm{L})$ & 1 \\
& $2-3 \mathrm{mg} / \mathrm{dL}(34.2-51.3 \mu \mathrm{mol} / \mathrm{L})$ & 2 \\
Albumin & $>3 \mathrm{mg} / \mathrm{dL}(>51.3 \mu \mathrm{mol} / \mathrm{L})$ & 3 \\
& $>3.5 \mathrm{~g} / \mathrm{dL}(>35 \mathrm{~g} / \mathrm{L})$ & 1 \\
& $2.8-3.5 \mathrm{~g} / \mathrm{dL}(28-35 \mathrm{~g} / \mathrm{L})$ & 2 \\
International normalized ratio & $<2.8 \mathrm{~g} / \mathrm{dL}(<28 \mathrm{~g} / \mathrm{L})$ & 3 \\
& $<1.7$ & 1 \\
Ascites & $1.7-2.2$ & 2 \\
& $>2.2$ & 3 \\
& Absent & 1 \\
Slight & Moderate & 2 \\
& No encephalopathy & 3 \\
& Grade $1-2$ & 1 \\
& Grade $3-4$ & 2 \\
\hline
\end{tabular}

\section{References}

32. Kamath PS, Wiesner RH, Malinchoc M, Kremers W, Therneau TM, Kosberg CL, D'Amico G, Dickson ER, Kim WR. A model to predict survival in patients with end-stage liver disease. Hepatology 2001;33:464-70.

33. Kim WR, Biggins SW, Kremers WK, Wiesner RH, Kamath PS, Benson JT, Edwards E, Therneau TM. Hyponatremia and mortality among patients on the liver-transplant waiting list. N Engl J Med 2008;359:1018-26.

34. Child CG, Turcotte JG. Surgery and portal hypertension. Major Probl Clin Surg 1964;1:1-85. 
Table S1 Packages of R software used in this study

\begin{tabular}{lc}
\hline Functions & R package \\
\hline Harrell's C-index, log-rank & survival \\
PH-CR model & cmprsk \\
AUC & pROC \\
Bar diagrams and ROC & ggplot2 \\
IDI & survIDINRI \\
Hosmer-Lemeshow test & ResourceSelection \\
VIF & car \\
Kaplan-Meier & survminer \\
Calibration curves & rms \\
\hline
\end{tabular}

C-index, concordance index; $\mathrm{PH}-\mathrm{CR}$, proportional-hazards competing risk; $A \cup C$, area under the receiver operating characteristic curve; ROC, receiver operating characteristic; IDI, Integrated Discriminating Improvement; VIF, variance inflation factor.

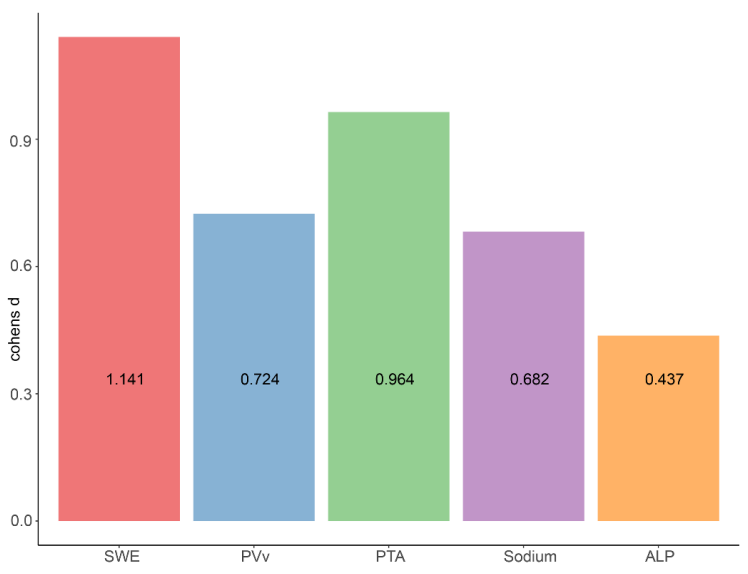

Figure S1 Percentage effect size (mean the difference between alive and dead patients/standard deviation) for the main predictors of 90-day ACLF development in univariate analysis. ACLF, acuteon-chronic liver failure; SWE, shear wave elastography; PVv, peak velocity of portal vein; PTA, prothrombin activity; ALP, alkaline phosphatase.
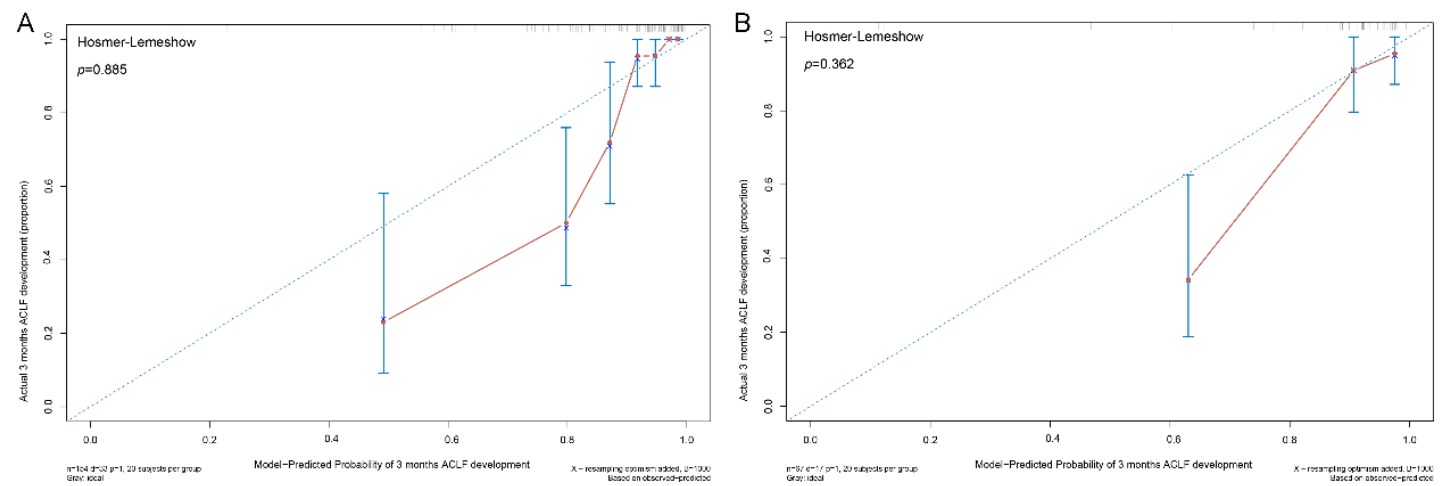

Figure S2 Calibration curves for estimation of 90-day ACLF development predicted by the SWE model in the derivation (A) and validation cohorts (B). ACLF, acute-on-chronic liver failure; SWE, shear wave elastography. 


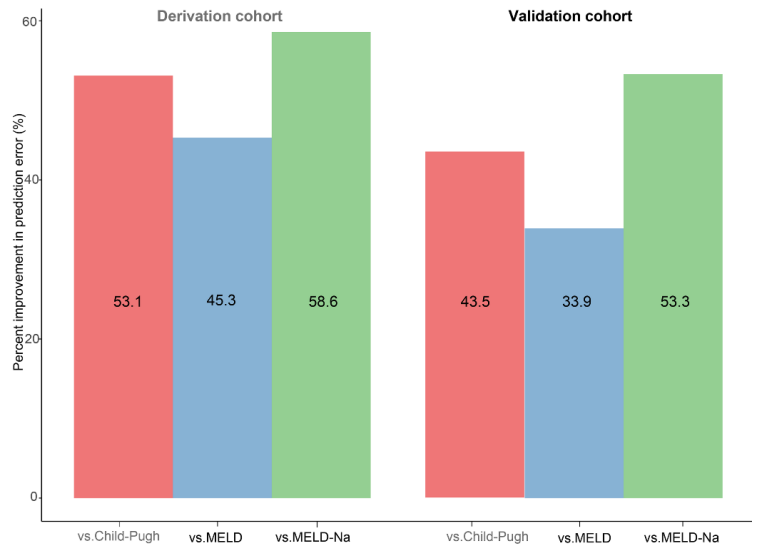

Figure S3 Relative (percentage) reduction in prediction error rates of the SWE model score compared to Child-Pugh, MELD, and MELD-Na scores. SWE, shear wave elastography; MELD, model for end-stage liver disease.

Table S2 Summary of baseline patient characteristics in the derivation and validation cohorts by 90-day ACLF development

\begin{tabular}{|c|c|c|c|c|c|c|}
\hline Characteristics & \multicolumn{3}{|c|}{ Derivation cohort } & \multicolumn{3}{|c|}{ Validation cohort } \\
\hline Age & $47.24 \pm 11.02$ & $52.03 \pm 12.33$ & 0.045 & $50.29 \pm 9.22$ & $55.92 \pm 12.25$ & 0.088 \\
\hline Female sex & $5(15.2)$ & $36(29.8)$ & 0.120 & $6(35.3)$ & $12(24.0)$ & 0.364 \\
\hline MBP & $88.80 \pm 12.66$ & $87.31 \pm 13.02$ & 0.558 & $81.45 \pm 10.20$ & $88.68 \pm 11.86$ & 0.028 \\
\hline Ascites & 29 (87.9) & $104(86.0)$ & 1.000 & $16(94.1)$ & $43(86.0)$ & 0.448 \\
\hline Splenomegaly* & $24(75.0)$ & $90(76.9)$ & 0.820 & $15(88.2)$ & $38(80.9)$ & 0.712 \\
\hline \multicolumn{7}{|l|}{ Laboratory data } \\
\hline HBeAg: positive & $14(42.4)$ & $38(31.4)$ & 0.235 & $5(29.4)$ & $15(30.0)$ & 0.963 \\
\hline Log HBV DNA, copies/mL & $5.62(4.26-7.29)$ & $5.06(2.93-6.48)$ & 0.193 & $5.76(4.71-7.16)$ & $4.54(2.93-5.76)$ & 0.149 \\
\hline White-cell count, $\times 10^{9} / \mathrm{L}$ & $5.56(4.43-7.27)$ & $4.35(2.97-5.92)$ & 0.016 & $5.55(1.96-6.76)$ & $4.61(3.44-7.42)$ & 0.795 \\
\hline PLT, $10^{9} / \mathrm{L}$ & $85.0(59.0-112.0)$ & $91.0(49.0-132.0)$ & 0.814 & $67.0(44.0-122.0)$ & $93.0(53.5-163.0)$ & 0.252 \\
\hline Serum albumin, g/dL & $2.82 \pm 0.52$ & $2.91 \pm 0.54$ & 0.392 & $3.00 \pm 0.63$ & $2.95 \pm 0.52$ & 0.718 \\
\hline Serum bilirubin, mg/dL & $12.54(10.14-17.29)$ & $4.49(3.08-11.85)$ & $<0.001$ & $10.97(3.82-18.65)$ & $4.45(2.84-15.17)$ & 0.103 \\
\hline
\end{tabular}

Table S2 (continued) 
Table S2 (continued)

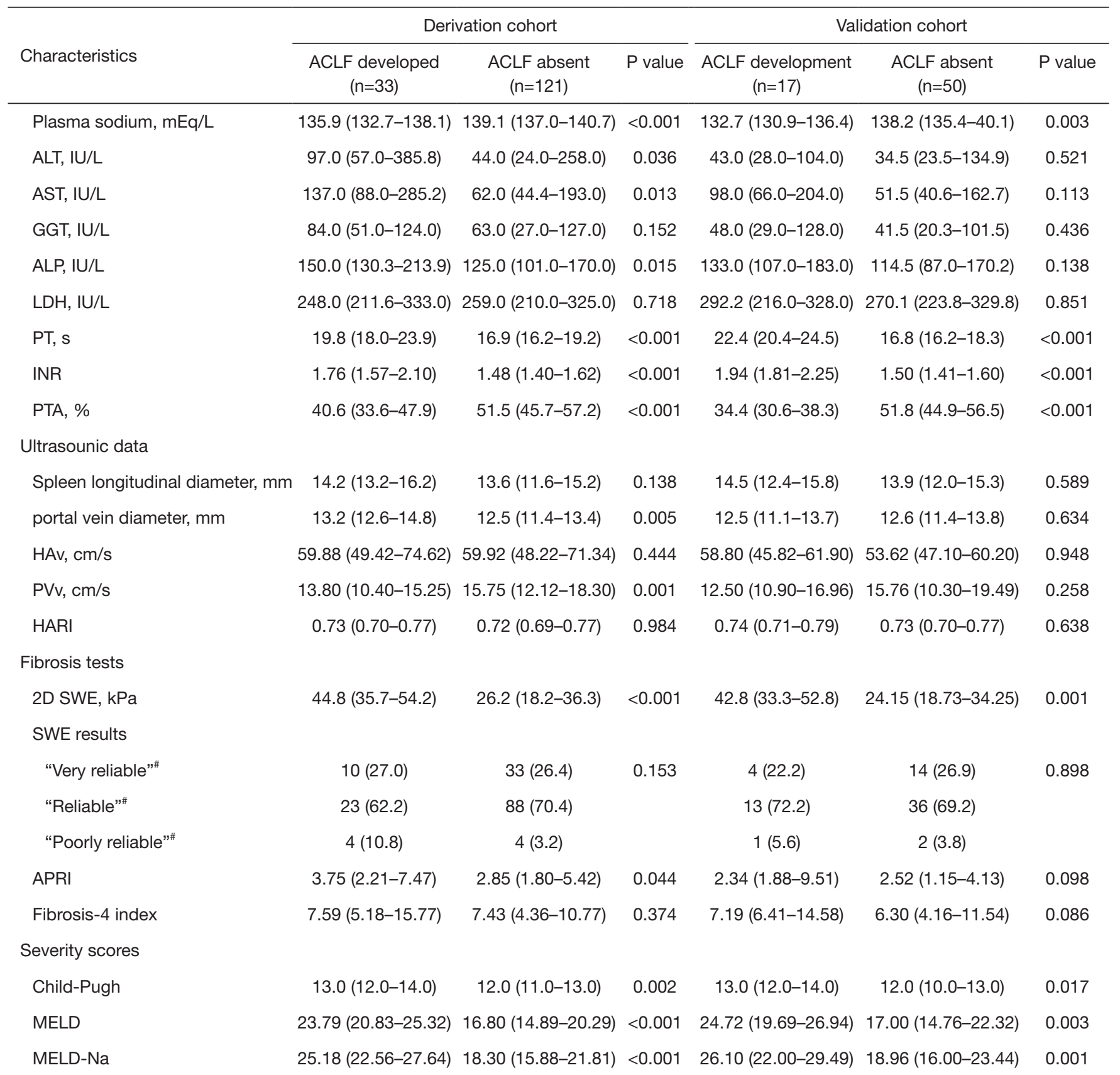

Outcome

90-day mortality rate ${ }^{\dagger}$

16 (48.5)

5 (4.1)

8 (47.1)

2 (4.0)

Continuous data were expressed as mean \pm standard deviation or median (25-75\% quantiles); categorical data were expressed as $\mathrm{n}$ (\%). *, 1 patients ACLF developed and 4 patients ACLF absent of the derivation cohort and 3 patients ACLF absent of the validation cohort underwent splenectomy; ", 2D SWE reliability by IQR/M: "very reliable" (IQR/M $\leq 0.10)$, "reliable" $(0.10<\mathrm{IQR} / \mathrm{M} \leq 0.3)$, and "poorly reliable" $(\mathrm{IQR} / \mathrm{M}>0.30) ;{ }^{\dagger}$, the start date of the follow-up period was the date of hospital admitted. ACLF, acute-on-chronic liver failure; MBP, mean arterial pressure; HBeAg, hepatitis B e antigen; HBV, hepatitis B viral; PLT, platelet count; ALT, alanine aminotransferase; AST, aspartate aminotransferase; GGT, g-glutamyl transferase; ALP, alkaline phosphatase; LDH, lactate dehydrogenase; PT, prothrombin time; INR, international normalized ratio; PTA, prothrombin activity; HAv, hepatic arterial velocity; PVv, peak velocity of portal vein; HARI, hepatic arterial resistive index; 2D, two-dimensional; SWE, shear wave elastography; APRI, aspartate aminotransferase-to-platelet ratio index; MELD, model for end-stage liver disease; IQR/M, interquartile range/median ratio. 